wäckerlin, C., Siewert, D., Jung, T. A., \& Ballav, N. (2013). On-surface coordination chemistry: Direct imaging of the conformational freedom of an axial 1igand at room temperature. Physical Chemistry Chemical Physics, 15(39), 16510-16514. https://doi.org/10.1039/c3cp50966d

\title{
On-Surface coordination chemistry: Direct imaging of the conformational freedom of an axial ligand at room-temperature
}

\author{
Christian Wäckerlin*, Dorota Siewert ${ }^{*}$ Thomas A. Jung*, Nirmalya Ballav* \\ Received (in XXX, XXX) Xth XXXXXXXXX 20XX, Accepted Xth XXXXXXXXX 20XX \\ ${ }_{5}$ DOI: $10.1039 / \mathrm{b000000x}$
}

The on-surface ligation of nitric oxide (NO) with Cotetraphenylporpyrin (CoTPP) sublimed onto oxygenreconstructed $\mathrm{Ni}(001)$ is studied by room-temperature scanning tunneling microscopy (STM) and complementary

${ }_{10}$ photoemission spectroscopies. On the oxygen-reconstructed substrates, the porphyrines are observed to form wellordered, self-assembled layers. STM directly images the NO ligand as a characteristic feature in the center of the molecule. At certain STM imaging conditions the dynamicity 15 of this feature can be related to the temperature-activated conformational flexibility of the NO ligand. This provides an indirect confirmation for the bending of the Co-NO bond, as predicted from coordination chemistry.

On-surface coordination-chemistry involves the study of metal 20 coordination at the surface which provides an environment which is at times modifying at other times not. For the reason of these differences and for the fact that on the surface many experiments - in particular by imaging or adressing of single reactants - are possible which are not possible in the liquid or 25 gas phase - this field is rapidly emerging. Apart from the now well-established use of coordinative-bonds to form stable 2D metal-organic layers ${ }^{1}$ and on-surface synthesis ${ }^{2-4}$, the axialcoordination of a ligand onto the free site of square-planar complexes is of great interest: axial coordination allows to 30 control the electronic ${ }^{5,6}$ and magnetic properties $^{7-9}$ of single transition-metal ions as well as to control chemical reactivity as used in catalysis ${ }^{10}$ and - embedded in protein architectures - in biochemistry ${ }^{11}$.

In this report we present studies about the on-surface 35 coordination of nitric oxide (NO) with Cotetraphenylporphyrin (CoTPP). In contrast to other work ${ }^{5,12}$, we study the on-surface coordination chemistry on oxygenreconstructed $(\mathrm{O}-\mathrm{c}(2 \times 2))$ nickel films $(\mathrm{Ni}(001))$ grown on copper $(\mathrm{Cu}(001))$ single crystals (cf. illustration in Fig.1a).

40 This setup is motivated by the modified physical and chemical interaction between the surface support and the ad-molecule. We here focus on the on-surface coordination-chemistry while in earlier studies we reported on the modification of the exchange coupling between CoTPP and magnetic substrates. ${ }_{45}$ The axial ligation of CoTPP (one unpaired electron in $\mathrm{d}_{\mathrm{z}}{ }^{2}$ ) with NO (one unpaired electron in $\pi^{*}$ ) has been studied in bulk / solution $^{13,14}$ as well as in the on-surface environment. ${ }^{5,7,8,12}$ For the $\sigma$-type overlap between the $\mathrm{d}_{\mathrm{z}}{ }^{2}$ orbital of Co and the $\pi^{*}$ of NO molecular orbital (MO) theory 50 predicts a $\sim 120^{\circ} \mathrm{Co}-\mathrm{NO}$ bond-angle. The coordination of NOligands onto CoTPP on $\mathrm{Ag}(111)$ has been studied earlier by low-temperature STM at $6 \mathrm{~K}$ and has been compared with $\mathrm{CO}$ exposed CoTPP - where cis-(CO) $)_{2}$-CoTPP is observed. ${ }^{12,15}$ In our study, the CoTPP-nitrosyl complexes are imaged at room55 temperature, in contrast. This allows us to directly observe the conformational libration of the $\sim 120^{\circ}$-bent NO-ligand, which is imaged as streaks surrounding the binding site in the center of the porphyrins (vide infra). Furthermore, CoTPP/Ag(111) ${ }^{16}$ is in the saddle-shape conformation, whereas the molecule is 60 adsorbed in flat geometry on $\mathrm{O} / \mathrm{Ni}$ (vide infra).

The substrate preparation is analogous to oxygen reconstructed cobalt films and is described elsewhere. ${ }^{17}$ The scanning tunneling micrscopy (STM) data presented here is aquired at room temperature (RT). Positive bias voltages ${ }_{65}$ result in electron tunneling from the tip into unoccupied states of the sample. In previous studies, we have observed that porphyrines / pthalocyanines form self-assembled layers on oxygen-reconstruced $\mathrm{Cu}(001)$ and $\mathrm{Co}(001)$ substrates. In contrast these molecules appear randomly arranged when 70 adsorbed on native (clean) $\mathrm{Cu}(001)$ and $\mathrm{Co}(001)$ substrates. ${ }^{17,18,7-9,4}$ This reflects that self-assembly is facilitated by the lower chemical reactivity of the oxygenreconstructed substrate which allows for the nessecary molecular diffusion. The fact that pthalocyanines on ${ }_{75} \mathrm{O} / \mathrm{Co}(001)$ were imaged with very high intra-molecular resolution $^{9}$ suggests the oxygen-reconstruction serves a similar - even though maybe weaker than alkali-halide carpet layers- role in decoupling the molecular adsorbates and their orbitals from metallic substrates. ${ }^{19}$

${ }_{80}$ Figures 1b\&c show STM images of the oxygen reconstructed $\mathrm{Ni}(\mathrm{O} / \mathrm{Ni}(001))$ substrate. The $\mathrm{O} / \mathrm{Ni}(001)$ adlayer reconstructs the surface to form terraces with rectangular edges running exactly along the $\mathrm{Cu}(001)$ principal directions (see Fig. 1b). ${ }^{20}$ This topography is characteristically different from the pure 85 metallic $\mathrm{Ni}(001)$ film ${ }^{20,7}$ and resembles oxygen recontructed $\mathrm{Co}(001) .{ }^{17}$ In Fig. 1c a step edge of the $\mathrm{O} / \mathrm{Ni}(001)$ film is shown with atomic resolution. It is important to note that one should take special care while assigning the molecular features in an STM image: in Supporting Information we 90 present STM data where the molecules resemble stars and their lobe assignment is compromsed by a tip-artifact. 

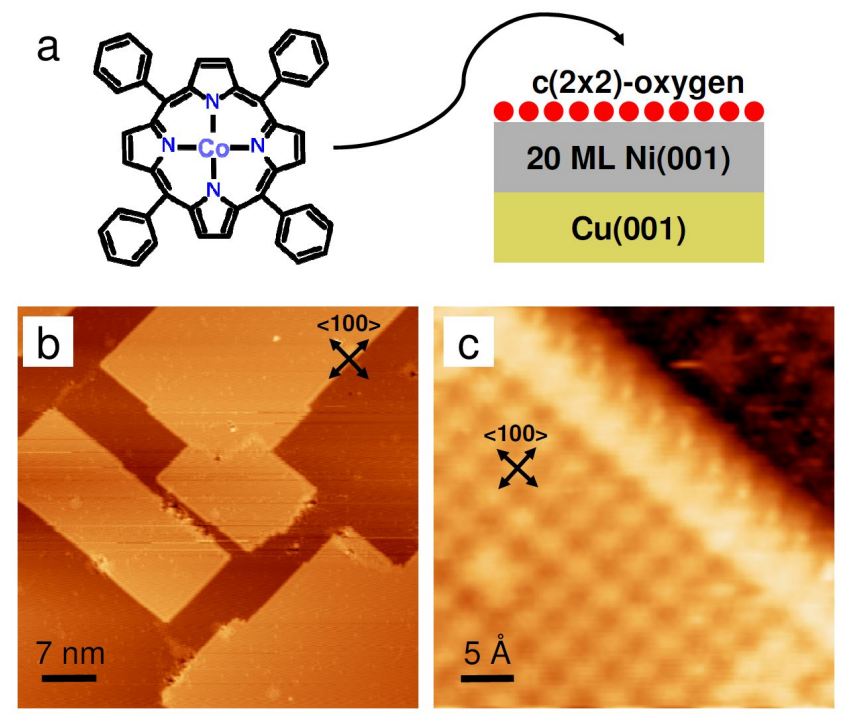

Fig. 1 (a) Molecular scheme of Co-tetraphenylporphyrin (CoTPP) and sketch of the sample composition: CoTPP is sublimed onto an oxygen-reconstructed $\mathrm{Ni}$ thin film grown on a $\mathrm{Cu}(001)$ single crystal. 5 (b) and (c) STM data of the oxygen-reconstructed Ni film (denoted $\mathrm{O} / \mathrm{Ni}(001)$ ): an overview and atomic resolution, respectively. The arrows indicate $\mathrm{Cu}(001)$ principal directions. All experiments are performed at room temperature.

${ }_{10}$ The such prepared surface serves as a substrate for the second step of the experiment, the CoTPP evaporation. The evaporation was performed from a home-built evaporator onto the $\mathrm{O} / \mathrm{Ni}(001)$ substrate kept at $\mathrm{RT}$. The molecular coverage was monitored by quartz crystal microbalance and cross15 checked by X-ray photoelectron spectroscopy (XPS) and STM. Fig. 2a shows an STM image of a CoTPP monolayer with some symbolic molecules superimposed onto the data. The molecules on $\mathrm{O} / \mathrm{Ni}(001)$ assemble in a chiral arrangement similar to the one presented on $\mathrm{O} / \mathrm{Co}(001) .{ }^{17}$ The observed flat 20 conformation is consistent with reports on metal-TPPs on other square metal(001) surfaces. ${ }^{21,17}$ Two mirror domains taking an angle of $28^{\circ}$ with respect to each other can be found in large-scale STM images (data not shown). The selfassembled layer is formed by intermolecular interactions 25 between the phenyl residues and the porphyrin macrocyle. This supramolecular arrangement exhibits a square unit-cell of $\sim 1.29 \mathrm{~nm}$, similarly to other poprhyrins on fcc(001) substrates ${ }^{17}$. In the middle of the molecules, several darker spots are visible (Fig. 2a). Tentatively we associate these 30 defects to a minority component in the crucible: metal-free H2TPP, which, upon reaction with oxygen reconstructed substrate at RT, have self-metalated to form NiTPP. ${ }^{4}$ Fig. $2 b$ shows a phase shift domain boundary between two domains of the $2 \mathrm{D}$ self-assembled layers. Here, the distance between two 35 molecules is increased and the intermolecular interaction occurs between the phenyl rings of the neighbouring molecules. Additionally, two molecules of a second layer can be distinguished in Fig. 2a (cf. zoom-in in Fig. 2c). Interestingly, they appear in the flat conformation and lie 40 exactly on top ${ }^{22}$ of the molecule of the first layer.
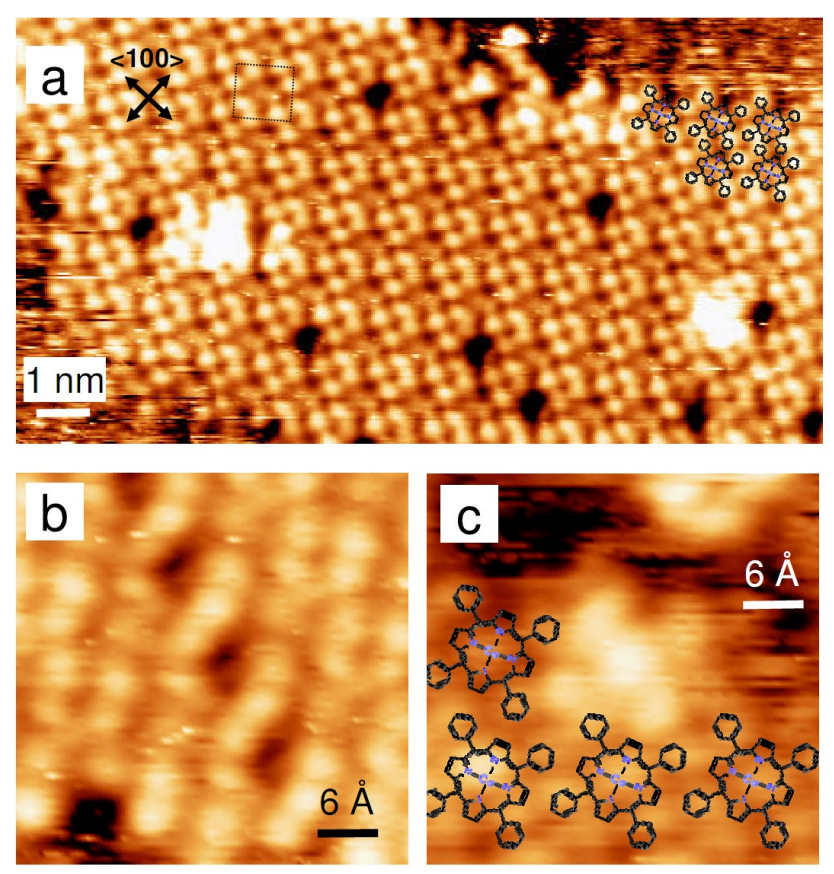

Fig. 2 STM data of $\sim 1$ ML of CoTPP on O/Ni(001). (a) An overview of the molecular lattice. The dotted rectangle markss the unit-cell of the lattice. A set of molecular schemes is superimposed onto part of 45 the lattice to guide the eye. (b) High resolution image of a phase shift domain boundary between two supramolecular islands. (c) CoTPP molecule imaged by STM in the second monolayer. The detailed analysis of the registry reveals that the $2^{\text {nd }}$ layer molecules stack in the 'on-top' position.

50

In the final step of the experiment we have exposed a monolayer of CoTPP/O/Ni(001) to 6000 Langmuir $\left(\mathrm{p}=8^{*} 10^{-6}\right.$ mbar) of nitric oxide (NO) and recorded a set of STM data (see Figs. 3 and 4). Upon NO exposure, the NO ligand binds 55 to the Co-porphyrin via its open top site. ${ }^{5,7,8,12} \mathrm{We}$ were able to image the NO-CoTPP complexes at RT at different biasvoltages. In Fig. $3 \mathrm{a}\left(\mathrm{U}_{\text {bias }}=2.0 \mathrm{~V}\right)$ the axially bonded NO ligand is imaged as regularly appearing streaky zones in the pattern of the molecules. Note that the porphyrins are a bit ${ }_{60}$ tricky to be identified in the patterns: around a molecular vacancy (blue square zoom-in) the imaged features can be assigned to the molecular structure. The molecules' 2D arrangement remains approximately the same after the NO exposure. The size of the squarely symmetric unit cell is ${ }_{65} \sim 1.30 \mathrm{~nm}$, which is virtually the same as before NO coordination. While there are no indications for a molecular rearrangement upon NO exposure on the oxygenreconstructed substrate, however this does not exclude the potential presence of NO adsorbed in-between the 70 porphyrines. Notably our result differs from earlier observations: NO exposure to CoTPP on $\operatorname{Ag}(111)$ was found to rearrange the unit-cell of the $2 \mathrm{D}$ self-assembled layer by being incorporated inbetween the CoTPP molecules. ${ }^{24}$ It seems possible that this different behaviour is due to the 75 different properties of the substrate-adsorbate systems: i) $\mathrm{Ag}(111)$ and $\mathrm{O} / \mathrm{Ni}(001)$ differ in the symmetry of the surface lattice, i.e. hexagonal vs. quadratic ii) the modified molecule- 
substrate interaction; this is reflected in the conformation of the CoTPP, either saddle-shaped or flat on $\operatorname{Ag}(111)$ and $\mathrm{O} / \mathrm{Ni}(001)$, respectively and iii) the lower and higher electronic corrugation of $\mathrm{Ag}(111)$ and $\mathrm{O} / \mathrm{Ni}(001)$, 5 respectively.
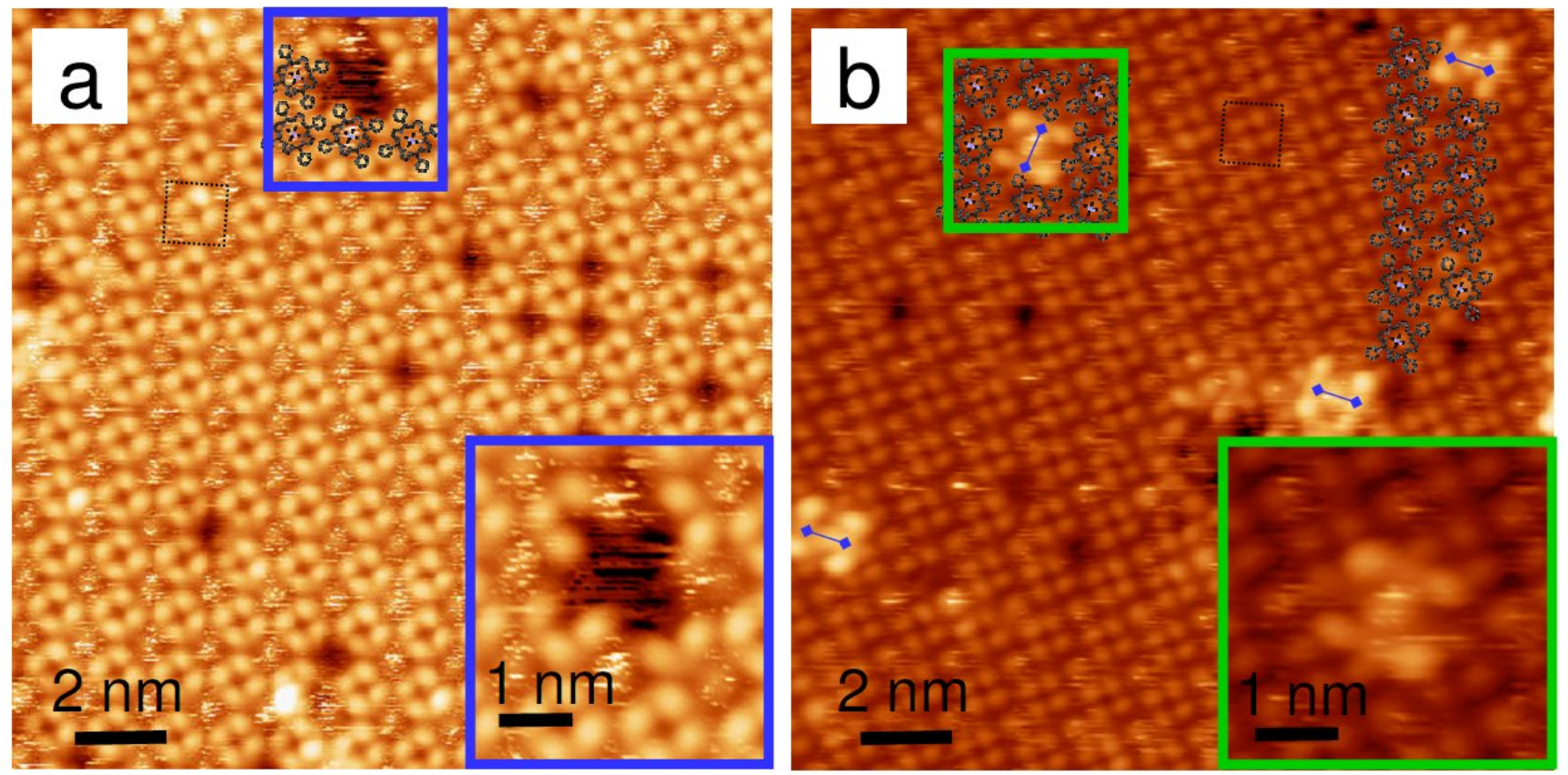

Fig. 3 STM data of the CoTPP monolayer on O/Ni(001) after exposure with NO (6000 Langmuir). (a) The CoTPP molecular lattice image is depicted well in the STM data acquired with $\mathrm{U}_{\text {bias }}=2.0 \mathrm{~V}$. The dotted square assigns the molecular unit-cell. The streaks appearing regularly at 10 the molecular centers are caused by the NO adsorbed at the Co-center metal atom of CoTPP and by its conformational libration at room temperature. Hollow sites have been assigned to Ni-metalated H2TPP molecules (see text). Single porphyrins can be identified around a molecular vacancy (blue square, cf. zoom-in on the right-hand side). (b) The same molecular lattice recorded with $U_{\text {bias }}=1.2 \mathrm{~V}$. The regularly appearing streaks are still distinguishable. Four molecules in the second layer are depicted by blue lines (cf. zoom-in section in the green square). 

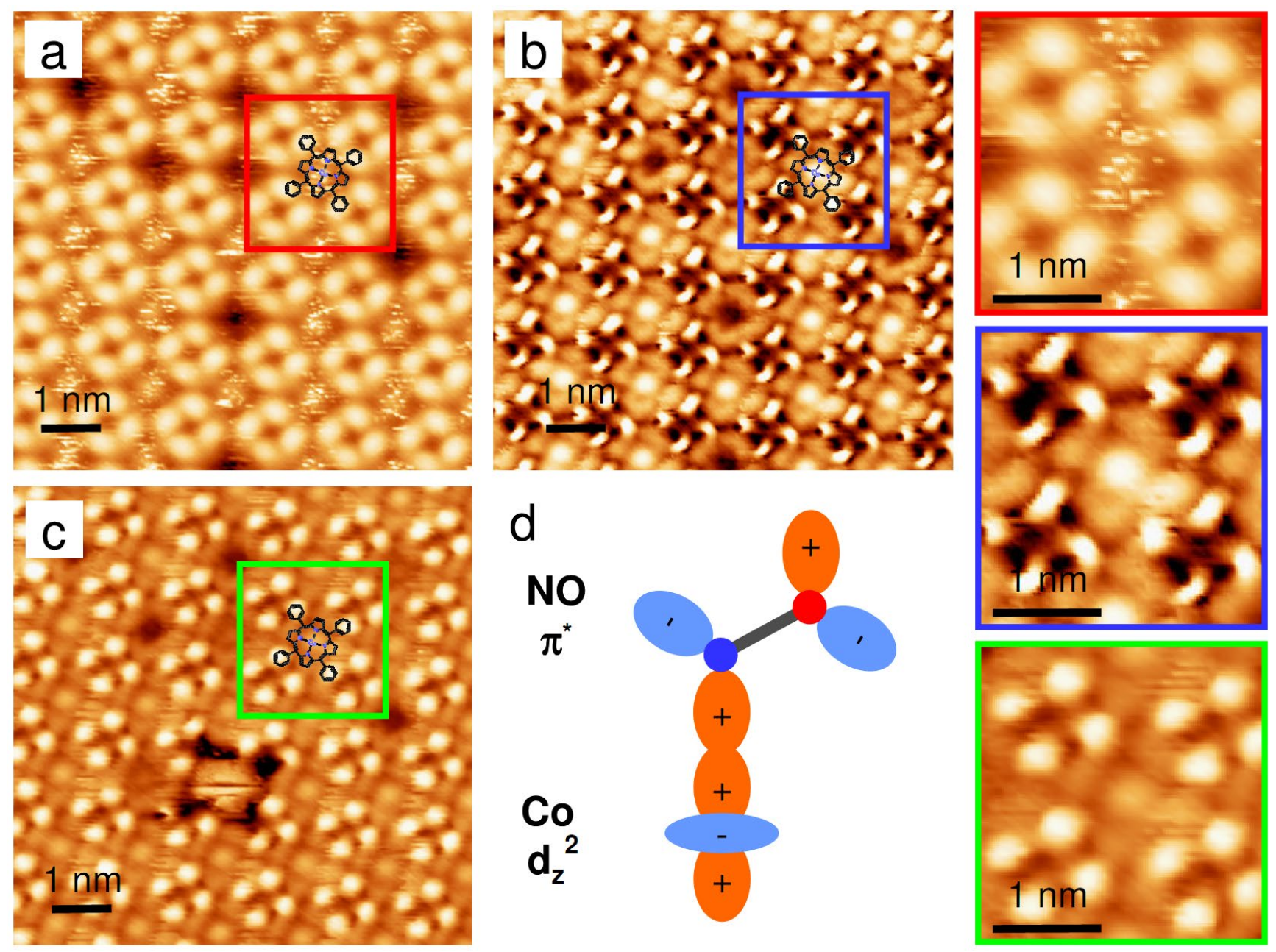

Fig. 4 Bias-dependent STM data of NO-exposed CoTPP on O/Ni. All three images display the same area on the sample. (a) U bias $=2.0$ V, the protrusions denote phenyl rings, $\mathrm{NO}$ visible as streaks in the molecular centres. Hollow sites are either NiTPP, or CoTPP without NO (b) $\mathrm{U}_{\text {bias }}=$ $0.3 \mathrm{~V}$, the protrusions denote pyrrole rings, the bright stripes (arranged in a windmill-shape) - phenyl rings. Bright molecular centres are NO 5 coordinated Co atoms, semi-dark molecular centres are Co without NO and dark ones NiTPP. The streaks now appear now slightly below the molecular centres. (c) $\mathrm{U}_{\text {bias }}=-0.5 \mathrm{~V}$, bright protrusions indicate phenyl rings, contrasts of molecular centres and NO molecules are the same as in (b). (d) The proposed Co-porphyrin NO $\sigma$-bond based on the overlap of the $\mathrm{Co}_{\mathrm{z}}{ }^{2}$ and NO $\pi^{*}$ orbitals. ${ }^{25}$ In c), one porphyrin has been removed from the lattice due to the interaction with the STM tip .

${ }_{10}$ When imaging with modified bias voltage (Fig. $2 \mathrm{~b}, \mathrm{U}_{\text {bias }}=$ $1.2 \mathrm{~V})$ CoTPP molecules appear differently, due to other molecular orbitals being probed (compare Fig. 4). Also the streaks of the imaged NO-ligands are here much less pronounced. Interestingly, the molecules of the second 15 monolayer (green zoom-in) again adsorb exactly on top of the first-layer, but here they are distorted and are found in the saddle-shape conformation.

The observation of individual ad-molecules with intramolecular resolution at RT is quite remarkable: to the 20 best of our knowledge so far only self-assembled ad-layers and not inidividual ad-molecules have been imaged by STM. ${ }^{23}$
Since we can also image the ad-molecules without the NO ligand, we relate the surprising stability of the ad-molecule with the flat conformation of the underlying first monolayer.

25 As the molecular symmetry of the ad-molecules is lowered (2fold) with respect to the first monolayer (4-fold), they spontaneously arrange in two energetically equivalent directions as indicated by the blue lines (Fig. 4b). The admolecules are also NO-ligated. We do not know if the CoTPP30 molecule below the ad-molecule is also with NO-ligand, but since NO-ligation has been shown to proceed into molecular multilayers, ${ }^{5}$ this is very plausible. These considerations suggest two possible mechanisms for the observed two-fold 
symmetry of the NO-CoTPP ad-molecules: i) due to NOligation of the ad-molecule inducing the saddle-shape conformation, ii) due to the (possibly) present NO-ligand in the first layer which lifts up the ad-molecule and which allows 5 for symmetry reduction via electronic / steric effects. In both images (Fig. 3a and 3b) the Ni-metalated H2TPP's are visible with dark spots in the middle.

Fig. 4 displays a set of STM images of the same sample area of the CoTPP monolayer recorded at different bias voltages.

$10 \mathrm{In}$ Fig. $4 \mathrm{a}\left(\mathrm{U}_{\text {bias }}=2.0 \mathrm{~V}\right)$ phenyl rings appear as bright protrusions and the dark spots are either Ni-metalated H2TPP or non-coordinated CoTPP. Fig $4 \mathrm{~b}$ recorded at $\mathrm{U}_{\text {bias }}=0.3 \mathrm{~V}$ resolves the former ambiguity. Here we see three different contrasts of molecular centers: bright, semi-dark and dark, 15 which we respectively assign as NO-ligated CoTPP, CoTPP without NO-ligand and Ni-metalated H2TPP. In Fig. 4c acquired at $\mathrm{U}_{\text {bias }}=-0.5 \mathrm{~V}$ the centres have similar contrast to Fig. $4 \mathrm{~b}$ and the phenyl rings appear as bright protrusions, as in Fig 4a. Interestingly, one molecule in Fig. 4c has been 20 removed by an STM tip while scanning, which evidences the previously anticipated low molecule-substrate interaction ${ }^{18}$.

\section{Table 1 Peak areas from X-ray photoelectron spectra.} CoTPP/O/Ni CoTPP/O/Ni + NO peak (kcps eV) peak (kcps $\mathrm{eV})$

$\begin{array}{ccc}\mathrm{C} 1 \mathrm{~s} & 11.95 & 10.7 \\ \mathrm{~N} 1 \mathrm{~s} & 3.3 & 8.5 \\ \mathrm{O} 1 \mathrm{~s} & 15.3 & 26.4 \\ \mathrm{Cu} 2 \mathrm{p} & 124 & 98 \\ \mathrm{Ni} 2 \mathrm{p} & 1897 & 1743\end{array}$

${ }_{25}$ Table 1 displays peak areas of X-ray photoelectron spectra. After NO-exposure, the $\mathrm{O} 1 \mathrm{~s}$ and $\mathrm{N} 1 \mathrm{~s}$ signals are significantly increased. One the basis of 4 nitrogen atoms per porphyrin, the increased N1s signal corresponds to $\sim 6$ additional NO molecules per porphyrin. Tentatatively, we ascribe this to 4 $30 \mathrm{NO}$ molecules within the self-assembled CoTPP layer (appearing bright in Fig. 5b) and to one NO acting as axial ligand.

\section{Conclusions}

35 In summary, we have shown that on oxygen-reconstruced $\mathrm{Ni}(001)$ substrates CoTPP self-assembles into well-ordered molecular monolayers consisting of molecules in the flat conformation. This is in-contrast to CoTPP on $\operatorname{Ag}(111)^{16}$, which adsorbs in saddle-shape conformation. We show that 40 the axial-NO ligand can be directly imaged even at roomtemperature and not only at $6 \mathrm{~K} .{ }^{12,6}$ The bias dependent roomtemperature images reveal the conformational flexibility of the NO ligand which provides an indirect confirmation for a bent $\mathrm{Co}-\mathrm{NO}$ bond. ${ }^{13,14}$ Interestingly, the CoTPP ad-molecules

45 observed in the second layer posses a lowered (2-fold) symmerty - however only after NO-ligation. Remarkably the present scanning tunneling microscopy study allows for the analysis of complex on-surface coordination reactions and monitors conformational and structural issues - even in ${ }_{50}$ dependence of first vs. second layer position of the molecules. This brings this method far beyond what has been achieved with spatially averaging spectroscopic methods while the latter method provides higher precision spectroscopic information which motivates further studies, in particular by 55 spectro-microscopy correlation.

\section{Acknowledgements}

This work was supported by the Swiss Nanoscience Institute (SNI), National Centre of Competence in Research Nanosciences (NCCR-Nano), Swiss National Science ${ }_{60}$ Foundation (SNSF), Holcim Foundation for the Advancement of Scientific Research, Switzerland. The authors sincerely thank Rolf Schelldorfer for technical support.

${ }^{a}$ Laboratory for Micro- and Nanotechnology, Paul Scherrer Institute, 5232 65 Villigen-PSI, Switzerland, E-Mail: thomas.jung@psi.ch

${ }^{b}$ Department of Physics, University of Basel, 4056 Basel, Switzerland ${ }^{\mathrm{c}}$ Department of Chemistry, Indian Institute of Science Education and Research (IISER), Pune - 411008, India, E-Mail: nballav@iiserpune.ac.in *To whom corresponence should be adressed.

$70 \$$ These authors contributed equally.

\section{Notes and references}

1. J. V. Barth, Surf. Sci., 2009, 603, 1533-1541.

2. J. M. Gottfried, K. Flechtner, A. Kretschmann, T. Lukasczyk, and H.-P. Steinrück, J. Am. Chem. Soc., 2006, 128, 5644-5645.

3.75 W. Auwärter, A. Weber-Bargioni, S. Brink, A. Riemann, A. Schiffrin, M. Ruben, and J. V. Barth, ChemPhysChem, 2007, 8, 250-254.

4. J. Nowakowski, C. Wäckerlin, J. Girovsky, D. Siewert, T. A. Jung, and N. R. Avery, Chem. Commun., 2013, 49, 2347-2349.

5. K. Flechtner, A. Kretschmann, H.-P. Steinrück, and J. M. Gottfried, J. 80 Am. Chem. Soc., 2007, 129, 12110-12111.

6. W. Hieringer, K. Flechtner, A. Kretschmann, K. Seufert, W. Auwärter, J. V. Barth, A. Görling, H.-P. Steinrück, and J. M. Gottfried, J. Am. Chem. Soc., 2011, 133, 6206-6222.

7. C. Wäckerlin, D. Chylarecka, A. Kleibert, K. Müller, C. Iacovita, F. 85 Nolting, T. A. Jung, and N. Ballav, Nat. Commun., 2010, 1, 61.

8. C. Wäckerlin, K. Tarafder, D. Siewert, J. Girovsky, T. Hählen, C. Iacovita, A. Kleibert, F. Nolting, T. A. Jung, P. M. Oppeneer, and N. Ballav, Chem. Sci., 2012, 3, 3154-3160.

9. C. Wäckerlin, J. Nowakowski, S.-X. Liu, M. Jaggi, D. Siewert, J. 90 Girovsky, A. Shchyrba, T. Hählen, A. Kleibert, P. M. Oppeneer, F. Nolting, S. Decurtins, T. A. Jung, and N. Ballav, Advanced Materials, 2013.

10. F. Sedona, M. Di Marino, D. Forrer, A. Vittadini, M. Casarin, A. Cossaro, L. Floreano, A. Verdini, and M. Sambi, Nat. Mater., 2012, 11, 970-977.

1 bs J. M. Berg, J. L. Tymoczko, and L. Stryer, Biochemistry, W. H. Freeman, New York, 2002.

12. K. Seufert, W. Auwärter, and J. V. Barth, J. Am. Chem. Soc., 2010, 132, 18141-18146.

13. W. R. Scheidt and J. L. Hoard, J. Am. Chem. Soc., 1973, 95, 8281-8288.

$14_{0}$ B. B. Wayland and J. V. Minkiewicz, J. Chem. Soc. Chem. Comm., 1976, 1015.

15. K. Seufert, M.-L. Bocquet, W. Auwärter, A. Weber-Bargioni, J. Reichert, N. Lorente, and J. V. Barth, Nat. Chem., 2011, 3, 114-119. 
16. W. Auwärter, K. Seufert, F. Klappenberger, J. Reichert, A. WeberBargioni, A. Verdini, D. Cvetko, M. Dell'Angela, L. Floreano, A. Cossaro, G. Bavdek, A. Morgante, A. P. Seitsonen, and J. V. Barth, Phys. Rev. B, 2010, 81, 245403.

175 D. Chylarecka, C. Wäckerlin, T. K. Kim, K. Müller, F. Nolting, A. Kleibert, N. Ballav, and T. A. Jung, J. Phys. Chem. Lett., 2010, 1, 14081413.

18. D. Chylarecka, T. K. Kim, K. Tarafder, K. Müller, K. Gödel, I. Czekaj, C. Wäckerlin, M. Cinchetti, M. E. Ali, C. Piamonteze, F. Schmitt, J.-P.

10 Wüstenberg, C. Ziegler, F. Nolting, M. Aeschlimann, P. M. Oppeneer, N. Ballav, and T. A. Jung, J. Phys. Chem. C, 2011, 115, 1295-1301.

19. J. Repp, G. Meyer, S. Stojković, A. Gourdon, and C. Joachim, Phys. Rev. Lett., 2005, 94, 026803.

20. R. Nünthel, T. Gleitsmann, P. Poulopoulos, A. Scherz, J. Lindner, E. 15 Kosubek, C. Litwinski, Z. Li, H. Wende, K. Baberschke, S. Stolbov, and T. . Rahman, Surf. Sci., 2003, 531, 53-67.

21. J. Brede, M. Linares, R. Lensen, A. E. Rowan, M. Funk, M. Bröring, G. Hoffmann, and R. Wiesendanger, Journal of Vacuum Science \& Technology B: Microelectronics and Nanometer Structures, 2009, 27, 20799.

22. M. Scarselli, P. Castrucci, D. Monti, and M. De Crescenzi, Surf. Sci., 2007, 601, 5526-5532.

23. F. Buchner, I. Kellner, W. Hieringer, A. Görling, H.-P. Steinrück, and H. Marbach, Phys. Chem. Chem. Phys., 2010, 12, 13082.

245 F. Buchner, K. Seufert, W. Auwärter, D. Heim, J. V. Barth, K. Flechtner, J. M. Gottfried, H.-P. Steinrück, and H. Marbach, ACS Nano, 2009, 3, 1789-1794.

25. T. Q. Nguyen, M. C.-S. Escaño, and H. Kasai, The Journal of Physical Chemistry B, 2010, 114, 10017-10021. 